\title{
Microsymposium
}

\section{Structural characterization of pseudo-dihyroorotase domain in yeast URA2}

Yujung Jeon $^{1}$, Ian Ross ${ }^{2}$, Michael Landsberg ${ }^{1}$, Ben Hankamer ${ }^{2}$, Bostjan Kobe ${ }^{1}$

${ }^{1}$ School Of Chemistry And Molecular Biosciences, The University Of Queesland, St Lucia, Australia, ${ }^{2}$ Institute for Molecular Bioscience, The University of Queensland, St Lucia, Australia

E-mail: yujung.jeon@uqconnect.edu.au

CAD is a large, multifunctional protein, composed of three enzymatic domains: carbamoyl phosphate synthase II (CPS II), aspartate transcarbamoylase (ATC), and dihydroorotase (DHO). The protein is involved in the three initial stages of de novo pyrimidine biosynthesis, required for the continuous production of pyrimidine nucleotides for living. In prokaryotes, the three enzymes are present in individual forms and acts independently, where as the three components are covalently linked together $(\sim 240 \mathrm{kDa})$ in eukaryotes. Since the up-regulation of the protein is often associated with the tumour cell development, the protein has been of an interest to many cancer biologists as a potential for anti-tumour drugs [1]. Up to date, not much structural information is available for CAD protein, other than that it forms a large homo-hexamer complex $(1.5 \mathrm{MDa})$ in the cellular environment [2]. As this assembly formation is thought to facilitate efficient molecular tunnel system in eukaryotic systems; our objective was to provide the structural evidences behind the oligomerisation of the CAD multienzyme complex.

Yeast (Saccharomyces cerevisiae) model was chosen for this study, as the organism produces URA2, an equivalent protein to CAD. URA2 is a bi-functional protein that is composed of CPS II and ATC, and pseudo-DHO (pDHO) domains. Though this defective $\mathrm{pDHO}$ domain lost its enzymatic function, the sequence is still homologous to other active DHO from CAD in other eukaryotic organisms [3]. The reason why the pDHO domain has not been eradicated for its inactivity is not yet known - our hypothesis was that the defective domain has an alternative role for full-length CAD/URA2 complex.

We successfully expressed and purified the yeast pDHO domain using E. coli system, crystallized and solved the crystal structure at high resolution $(1.2 \AA)$. Despite the similar overall structure to other active DHO enzymes, we were able to identify a number of different structural traits that accounts for the loss of the enzymatic activity from the yeast pDHO crystal structure. Biophysical studies, MALS and SAXS, identified the pDHO domain by itself is present in solution in the equilibrium state between two oligomeric forms, dimer and hexamer. While the hexamer formation occurred in concentration dependent manner, we found the co-expression of pDHO with the adjacent ATC domain successfully yielded a monomeric peak at the molecular weight corresponding to the hexamer size. Negative electron microscopy showed the pDHO-ATC protein forms the stable homogenous donut/disc shaped particles, suggesting the linked domains may act as a core scaffold for the hexamerisation of the full-length URA2/CAD. In summary, the results suggested and highlighted the pDHO domain and its interaction with the adjacent domains may have the potential structural role in forming homo-hexamer URA2 complex, despite the inactivity.

1. Evans, D.R. \& H.I. Guy. (2004). J Biol Chem, 279 (32), 33035-8.

2. Lee, L., et al. (1985). Proc Natl Acad Sci U S A, 82 (20), 6802-6.

[3] Hervé, G., et al. (1993). Biochem Soc Trans, 21 (1), 195-8.

Keywords: Crystallography, Electron microscopy, Enzymes 